and splicing-variant stoichiometry. This is particularly important if altering the expression levels of different transcripts results in highly heterogeneous phenotypes. Indeed, ectopic expression of VEGF can result in blood vessels with unpredictable properties, including hyperpermeability ${ }^{6}$.

Rebar et al. seem to have largely circumvented such problems with ectopic VEGF expression. They found that their adenoviral-delivered zinc-finger transcription factor induces expression of native VEGF isoforms leading to the production of an apparently physiologically normal vasculature (Fig. 1). Moreover, this vasculature was functional; compared with a control adenoviral reporter gene construct, the engineered transcription factor accelerated wound healing, which relies on angiogenesis.

The new study verifies the value of transcription factors as targets for drug development and highlights their potential to control angiogenesis in a therapeutic context. But two immediate questions remain. First, is this strategy applicable to other diseases that might benefit from angiogenesis induction, such as ischemia? Second, would it be possible to inhibit angiogenesis by turning off the transcription of pro-angiogenic molecules? Potent and selective gene suppression has already been achieved for the endogenous proto-oncogenes $E R B B-2$ and $E R B B-3$ in cell culture ${ }^{7}$. Moreover, present engineered transcrip- tion factors do better than RNA interference-another attention-grabbing technique-when it comes to silencing gene expression (although such approaches must still be compared side-by-side).

The potential to either activate or repress gene transcription is a major advantage of a transcription factor-based approach. Simply changing the effector domain fused to a zinc-finger protein can alter the protein's properties. For example, gene activation could be achieved by fusion of a targeted zinc-finger protein to an activation domain (such as VP-16), whereas repression could be achieved by fusing the same DNA-binding motif to a repression domain (such as the Kruppelassociated box (KRAB)). Additionally, transcription factors could be chemically modified allowing fine-tuning of gene activation or repression ${ }^{2,7}$.

De novo design of transcription factors with biological function is in its early stages. However, preclinical and clinical applications are certain to appear in the future. The low intrinsic toxicity of designed transcription factors in transgenic organisms further supports their clinical potential $^{5}$. Indeed, with proper design it should be possible to regulate multiple genes in a biosynthetic or developmental pathway with a single designed transcription factor. Artificial transcriptional factors might eventually be used to direct the formation of particular, desirable endothe- lial-cell phenotypes in blood vessels of tissues—or even whole organs ${ }^{8}$ - in order to artificially program protein-expression profiles within selective vascular beds. The work of Rebar et al. effectively sets the stage for these developments.

1. Rebar, E.J. et al. Induction of angiogenesis in a mouse model using engineered transcription factors. Nature Med. 8, 1427-1432 (2002).

2. Beerli, R.R. \& Barbas, C.F. III. Engineering polydactyl zinc-finger transcription factors. Nature Biotechnol. 20, 135-141 (2002).

3. Pabo, C.O., Peisach, E. \& Grant, R.A. Design and selection of novel Cys2His2 zinc finger proteins. Annu. Rev. Biochem. 70, 313-340 (2001).

4. Xu, L. et al. A versatile framework for the design of ligand-dependent, transgene-specific transcription factors. Mol. Ther. 3, 262-273 (2001).

5. Guan, X. et al. Heritable endogenous gene regulation in plants with designed polydactyl zinc finger transcription factors. Proc. Natl. Acad. Sci. USA 99, 13296-13301 (2002).

6. Thurston, G. et al. Angiopoietin-1 protects the adult vasculature against plasma leakage. Nature Med. 6, 460-463 (2000).

7. Beerli, R.R., Dreier, B. \& Barbas III, C.F. Positive and negative regulation of endogenous genes by designed transcription factors. Proc. Natl. Acad. Sci. USA 97, 1495-1500 (2000).

8. Pasqualini, R. \& Arap, W. Vascular targeting. in Encyclopedia of Cancer, Vol. 4, 2nd edn. (ed. Bertino, J.R.), 501-507 (Academic Press, San Diego-Oxford, 2002)

\section{${ }^{1}$ M.D. Anderson Cancer Center,}

The University of Texas Houston

Texas, USA

Email: rpasqual@mail.mdanderson.org

${ }^{2}$ The Scripps Research Institute,

La Jolla, California, USA

Email:carlos@scripps.edu

\title{
Breaking up the biofilm
}

The lungs of patients with cystic fibrosis can contain slimy biofilms of the bacterium Pseudomonas aeruginosa, enmeshed in thick airway mucus. These biofilms present a front against antibiotics and other treatments, and patients succumb to complications from such bacterial infections, often before their mid-30s.

Recent data have suggested that in the lung, biofilms persist under anaerobic conditions. In the October Developmental Cell, Sang Sun Yoon et al. describe experiments replicating these anaerobic

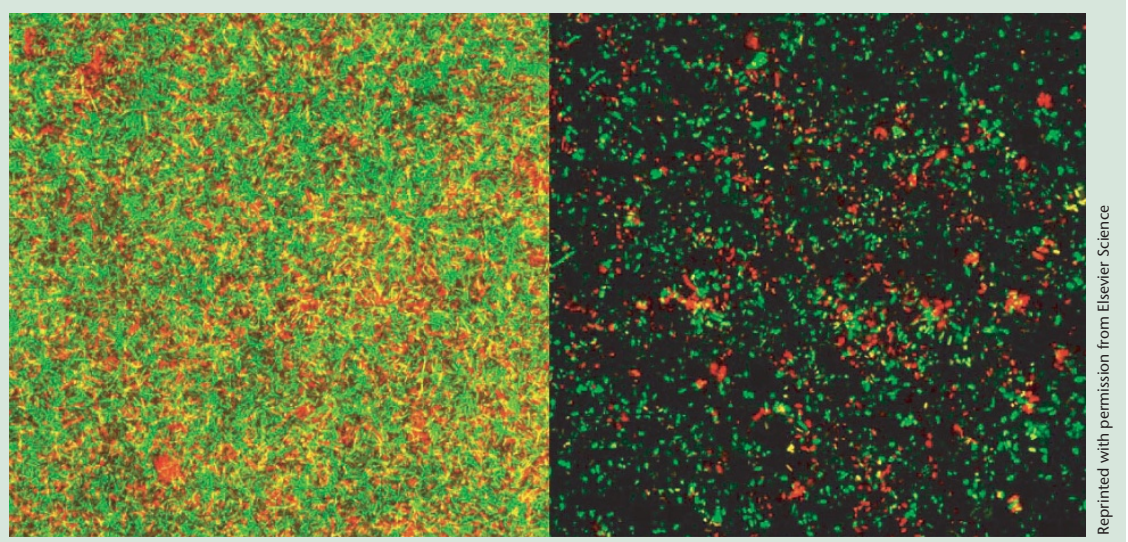
biofilms in culture. They find that $P$. aeruginosa form denser, more robust biofilms under anaerobic (left) than aerobic conditions (right). In both images, live bacteria are stained green and dead bacteria are red. The authors went on to identify genes that assist in biofilm formation in anaerobic conditions. Among these were the outer membrane protein $\mathrm{F}(\mathrm{OprF})$ gene, which was upregulated 5-fold during aerobic biofilm growth but 39-fold during anaerobic growth. Bacteria without OprF produced very poor anaerobic biofilms. Yoon et al. provide hints that bacteria lacking OprF, a channel-forming protein, are defective in a respiratory pathway critical for anaerobic growth.

Anaerobic conditions impair the effectiveness of many 'front-line' antibiotics such as tobramycin. If anaerobic biofilm formation could be effectively inhibited, say the authors, this might give these antibiotics a second chance to work in patients with particularly resilient $P$. aeruginosa populations. Indeed, vaccination with OprF has been shown to protect mice against $P$. aeruginosa infection.

CHARlotTe SCHUberT 